\title{
Suzuki - $F(\psi-\phi)-\alpha$ type fixed point theorem on quasi metric spaces
}

\author{
Venigalla Madhulatha Himabindu ${ }^{a}$ \\ ${ }^{a}$ Department of Mathematics, Koneru Lakshmaiah Educational Foundation, Vaddeswaram, Guntur - 522 502, \\ Andhra Pradesh, India.
}

\section{Abstract}

In this paper, we obtain a $\alpha$ - Suzuki fixed point theorem by using $C$ - class function on quasi metric spaces. Also we give an example which supports our main theorem.

Keywords: Quasi - metric space, Suzuki type contraction, $C$ - Class function, $\alpha$ - admissible mapping. 2010 MSC:

\section{Introduction}

In this paper $\mathbb{N}$ and $\mathbb{R}$ denote the sets of positive integers, respectively the set of real numbers, while $\mathbb{N}_{0}:=\mathbb{N} \cup\{0\}$ and $\mathbb{R}_{0}^{+}:=[0, \infty)$.

In 2008, the generalization theorem of Banach contraction principle [2], which was introduced by T.Suzuki [7, later this theorem is also referred as Suzuki type contraction. In 2014, Ansari [1] introduced the concept of $C$ - class functions and proved the unique fixed point theorems for certain contractive mappings with respect to the $C$ - class functions.

The aim of this paper is to prove a $\alpha$-Suzuki type fixed point theorem by using $(C)$ - class functions in quasi metric spaces.

\section{Preliminaries}

The aim of Suzuki [7] is to extend the well-known Edelstein's Theorem by using the notion of C-condition. Popescu [5] re-considered this approach to extend Bogin's fixed point theorem:

Email address: v.m.1.himabindu@gmail.com (Venigalla Madhulatha Himabindu) 
Theorem 2.1. Let a self-mapping $T$ on a complete metric space $(X, d)$ satisfies the following condition:

$$
\frac{1}{2} d(x, T x) \leq d(x, y)
$$

implies

$$
d(T x, T y) \leq a d(x, y)+b[d(x, T x)+d(y, T y)]+c[d(x, T y)+d(y, T x)]
$$

where $a \geq 0, b>0, c>0$ and $a+2 b+2 c=1$. Then $T$ has a unique fixed point.

First we recall some basic definitions which play crucial role in the theory of quasi metric spaces.

Definition 2.2. Let $X$ be a non-empty set $X$ and $q: X \times X \rightarrow R^{+}$be a function which satisfies: such that for all $x, y, z \in X$ :

$\left(q_{1}\right) q(x, y)=0$ if and only if $x=y$;

$\left(q_{2}\right) q(x, y) \leq q(x, z)+q(z, y)$.

The pair $(X, q)$ is called a quasi- metric space.

Example 2.3. Let $X=l_{1}$ be defined by

$$
l_{1}=\left\{\left\{x_{n}\right\}_{n \geq 1} \subset R, \sum_{n=1}^{\infty}\left|x_{n}\right|<\infty\right\} .
$$

Consider $d: X \times X \rightarrow[0, \infty)$ such that

$$
q(x, y)=\left\{\begin{array}{cl}
0 & \text { if } \preceq y \\
\sum_{n=1}^{\infty}\left|x_{n}\right| & \text { if } \succeq y
\end{array}\right.
$$

$q$ is a quasi-metric. Mention that $x \succeq y$ if $x_{n} \geq y_{n}$ for all $n$, where $x=\left\{x_{n}\right\}$ and $y=\left\{y_{n}\right\}$ are in $X$.

Definition 2.4. Let $(X, q)$ be a quasi-metric space.

$q(i)$ A sequence $\left\{x_{n}\right\}$ in $X$ is said to be convergent to $x$ if $\lim _{n \rightarrow \infty} q\left(x_{n}, x\right)=\lim _{n \rightarrow \infty} q\left(x, x_{n}\right)=0$.

q(ii) A sequence $\left\{x_{n}\right\}$ in $X$ is called left-Cauchy if for every $\epsilon>0$ there exists a positive integer $N=N(\epsilon)$ such that $q\left(x_{n}, x_{m}\right)<\epsilon$ for all $n \geq m>N$.

$q\left(\right.$ iii) A sequence $\left\{x_{n}\right\}$ in $X$ is called right-Cauchy if for every $\epsilon>0$ there exists a positive integer $N=N(\epsilon)$ such that $q\left(x_{n}, x_{m}\right)<\epsilon$ for all $m \geq n>N$.

$q(i v)$ A sequence $\left\{x_{n}\right\}$ in $X$ is called Cauchy sequence if for every $\epsilon>0$ there exists a positive integer $N=N(\epsilon)$ such that $q\left(x_{n}, x_{m}\right)<\epsilon$ for all $m, n>N$.

Remark: From definition it is obvious that a sequence $\left\{x_{n}\right\}$ in a quasi-metric space is Cauchy if and only if it is both left-Cauchy and right-Cauchy.

Ansari [1] introduced the concept of $C$-class functions as the following:

Definition 2.5. (See [1]) A mapping $F:[0,+\infty)^{2} \rightarrow R$ is called a $C$ - class function if it is continuous and for all $s, t \in[0,+\infty)$,

(a) $F(t, s) \leq s$

(b) $F(s, t)=s$ implies that either $s=0$ or $t=0$.

We denote $\mathcal{C}$ as the family of all $C$ - class functions. 
Example 2.6. (See [1]) The following functions $F:[0,+\infty)^{2} \rightarrow R$ are elements in $C$.

(1) $F(s, t)=s-t$ for all $s, t \in[0, \infty)$;

(2) $F(s, t)=m s$ for all $s, t \in[0, \infty)$ where $0<m<1$;

(3) $F(s, t)=\frac{s}{(1+t)^{r}}$ for all $s, t \in[0, \infty)$ where $r \in(0, \infty)$;

(4) $F(s, t)=(s+l)^{\frac{1}{(1+t)^{r}}}-l$ for all $s, t \in[0, \infty)$ where $l>1, r \in(0, \infty)$;

(5) $F(s, t)=s \log _{t+a} a$ for all $s, t \in[0, \infty)$ where $a>1$;

(6) $F(s, t)=s-\left(\frac{1+s}{2+s}\right)\left(\frac{t}{1+t}\right)$ for all $s, t \in[0, \infty)$;

(7) $F(s, t)=s \beta(s)$ for all $s, t \in[0, \infty)$ where $\beta:[0, \infty) \rightarrow[0,1)$ and is continuous;

(8) $F(s, t)=s-\varphi(s)$ for all $s, t \in[0, \infty)$ where $\varphi:[0, \infty) \rightarrow[0, \infty)$ is a continuous function such that $\varphi(t)=0$ if and only if $t=0$;

(9) $F(s, t)=\operatorname{sh}(s, t)$ for all $s, t \in[0, \infty)$ where $h:[0, \infty) \times[0, \infty) \rightarrow[0, \infty)$ is a continuous function such that $h(t, s)<1$ for all $s, t \in[0, \infty)$;

(10) $F(s, t)=s-\left(\frac{2+t}{1+t}\right) t$ for all $s, t \in[0, \infty)$;

(11) $F(s, t)=\sqrt[n]{\ln \left(1+s^{n}\right)}$ for all $s, t \in[0, \infty)$.

Definition 2.7. (See [1]) A function $\psi:[0, \infty) \rightarrow[0, \infty)$ is called an altering distance function if the following properties are satisfied:

(a) $\psi$ is nondecreasing and continuous;

(b) $\psi(t)=0$ if and only if $t=0$.

We denote $\Psi$ the family of all altering distance function.

Definition 2.8. (See [1]) A function $\varphi:[0, \infty) \rightarrow[0, \infty)$ is called an ultra altering distance function if the following properties are satisfied:

(a) $\varphi$ is continuous;

(b) $\varphi(t)>0$ for all $t>0$.

We denote $\Phi$ the family of all altering distance function.

In 2012, Samet et al. [6] introduced $\alpha$ - admissible mappings as the following:

Definition 2.9. (See. [6], [3] ) A mapping $f: X \rightarrow X$ is called $\alpha$-admissible if for all $x, y \in X$ we have

$$
\alpha(x, y) \geq 1 \Rightarrow \alpha(f x, f y) \geq 1
$$

where $\alpha: X \times X \rightarrow[0, \infty)$ is a given function.

Definition 2.10. [4] A mapping $f: X \rightarrow X$ is called a triangular $\alpha$-admissible if it is $\alpha$-admissible and satisfies

$$
\left.\begin{array}{l}
\alpha(x, y) \geq 1 \\
\alpha(y, z) \geq 1
\end{array}\right\} \Rightarrow \alpha(x, z) \geq 1
$$

where $x, y, z \in X$ and $\alpha: X \times X \rightarrow[0, \infty)$ is a given function.

Definition 2.11. [4] A mapping $f: X \rightarrow X$ is said to be weak triangular $\alpha$-admissible if it is $\alpha$-admissible and satisfies

$$
\alpha(x, f x) \geq 1 \Rightarrow \alpha\left(x, f^{2} x\right) \geq 1,
$$

where $\alpha: X \times X \rightarrow[0, \infty)$ is a given function.

Lemma 2.12. 4l Let $f: X \rightarrow X$ be a weak triangular $\alpha$-admissible mapping. Assume that there exists $x_{0} \in x$ such that $\alpha\left(x_{0}, f x_{0}\right) \geq 1$. If $x_{n}=f^{n} x_{0}$, then $\alpha\left(x_{m}, f x_{n}\right) \geq 1$ for all $m, n \in N_{0}$ with $m<n$.

The following auxiliary result is going to be used in the proof of existence theorems. 
Lemma 2.13. Let $f: X \rightarrow X$ be a triangular $\alpha$-admissible mapping. Assume that there exists $x_{0} \in X$ such that $\alpha\left(x_{0}, f x_{0}\right) \geq 1$ and $\alpha\left(f x_{0}, x_{0}\right) \geq 1$. If $x_{n}=f^{n} x_{0}$, then $\alpha\left(x_{m}, x_{n}\right) \geq 1$ for all $m, n \in N$.

Definition 2.14. Let $(X, q)$ be a quasi metric space and let $f: X \rightarrow X$ be a given mapping $f$ is an $F(\psi-\phi)-\alpha$-Suzuki- type rational contraction condition. If there exist two functions $\alpha: X \times X \rightarrow[0, \infty)$ such that $\alpha(x, y) \geq 1$ and

$$
\frac{1}{2} q(x, f x) \leq q(x, y)
$$

implies that

$$
\psi(q(f x, f y)) \leq F(\psi(M(x, y)), \varphi(M(x, y)))
$$

for all $x, y$ in $X$, where

$$
M(x, y)=\max \left\{q(x, y), \frac{1+q(x, f x) \cdot q(y, f y)}{1+q(x, y)}\right\}
$$

$\psi \in \Psi, \varphi \in \Phi$ and $F \in \mathcal{C}$.

Now we prove our main result.

\section{Main Results}

Theorem 3.1. Let $(X, q)$ be a complete quasi metric space and $f: X \rightarrow X$ be mappings such that $f$ is $F(\psi-\phi)-\alpha$-Suzuki- type rational contractive suppose that

(i) $f: X \rightarrow X$ is weak triangular $\alpha$-admissible mapping

(ii) there exists $x_{0} \in X$ such that $\alpha\left(x_{0}, f x_{0}\right) \geq 1$ and $\alpha\left(f x_{0}, x_{0}\right) \geq 1$

(iii) $f$ is continuous or If $\left\{x_{n}\right\}$ is a sequence in $X$ such that $\alpha\left(x_{n}, x_{n+1}\right) \geq 1$ and $\alpha\left(x_{n+1}, x_{n}\right) \geq 1$ for all $n$ and as $n \rightarrow \infty$, then there exists a subsequence $\left\{x_{n}(k)\right\}$ of $x_{n}$ such that $\alpha\left(x_{n}(k), x\right) \geq 1$ and $\alpha\left(x, x_{n}(k)\right) \geq 1$ for all $k$

Then $f$ has fixed point in $X$.

Proof. By assumption (ii), there exists $x_{0} \in X$, such that $\alpha\left(x_{0}, f x_{0}\right) \geq 1$ and $\alpha\left(f x_{0}, x_{0}\right) \geq 1$.

Define the sequence $\left\{x_{n}\right\}$ in $X$ as $f x_{n}=x_{n+1}, n=1,2,3, \cdots$

If $x_{n_{0}}=x_{n_{0}+1}$ for some $n_{0}>0$, then $x_{n_{0}}$ is a fixed point of $f$ and the proof is done. Assume that $x_{n} \neq x_{n+1}$ for all $n \geq 0$. Since $f$ is $\alpha$ - admissible,

$$
\alpha\left(x_{0}, f x_{0}\right)=\alpha\left(x_{0}, x_{1}\right) \geq 1 \Rightarrow \alpha\left(f x_{0}, f x_{1}\right)=\alpha\left(x_{1}, x_{2}\right) \geq 1
$$

and continuing we obtain

$$
\alpha\left(x_{n}, x_{n+1}\right) \geq 1 \text { forall } n \in N
$$

Since

$$
\frac{1}{2} q\left(x_{n}, f x_{n}\right) \leq q\left(x_{n}, x_{n+1}\right) .
$$

From (3), we get

$$
\begin{aligned}
& \psi\left(q\left(f x_{n}, f x_{n+1}\right)\right) \leq F\left(\psi\left(M\left(x_{n}, x_{n+1}\right)\right), \varphi\left(M\left(x_{n}, x_{n+1}\right)\right)\right) \\
& \begin{aligned}
M\left(x_{n}, x_{n+1}\right)= & \max \left\{q\left(x_{n}, x_{n+1}\right), \frac{1+q\left(x_{n}, x_{n+1}\right) \cdot q\left(x_{n+1}, x_{n+2}\right)}{1+q\left(x_{n}, x_{n+1}\right)}\right\} \\
= & \max \left\{q\left(x_{n}, x_{n+1}\right), q\left(x_{n+1}, x_{n+2}\right)\right\} .
\end{aligned}
\end{aligned}
$$


Hence,

$$
\psi\left(q\left(x_{n+1}, x_{n+2}\right)\right) \leq F\left(\psi\left(\max \left\{q\left(x_{n}, x_{n+1}\right), q\left(x_{n+1}, x_{n+2}\right)\right\}\right), \varphi\left(\max \left\{q\left(x_{n}, x_{n+1}\right), q\left(x_{n+1}, x_{n+2}\right)\right\}\right)\right) .
$$

If $q\left(x_{n+1}, x_{n+2}\right)$ is maximum then we have

$$
\psi\left(q\left(x_{n+1}, x_{n+2}\right)\right) \leq F\left(\psi\left(q\left(x_{n+1}, x_{n+2}\right)\right), \varphi\left(q\left(x_{n+1}, x_{n+2}\right)\right)\right)<\psi\left(q\left(x_{n+1}, x_{n+2}\right)\right)
$$

, which is a contradiction.

Hence $q\left(x_{n}, x_{n+1}\right)$ is maximum. Thus

$$
\psi\left(q\left(x_{n+1}, x_{n+2}\right)\right) \leq F\left(\psi\left(q\left(x_{n}, x_{n+1}\right)\right), \varphi\left(q\left(x_{n}, x_{n+1}\right)\right)\right.
$$

Since $\psi$ is increasing we have $q\left(x_{n+1}, x_{n+2}\right) \leq q\left(x_{n}, x_{n+1}\right)$.

Thus $\left\{q\left(x_{n}, x_{n+1}\right)\right\}$ is a non - increasing sequence of non - negative real numbers and must converge to a real number, say, $r \geq 0$. Suppose $r>0$.

Letting $n \rightarrow \infty$ in (4), we get $\psi(r) \leq F(\psi(r), \varphi(r))$. This implies that $\psi(r)=0$ and $\varphi(r)=0$ which yields

$$
\lim _{n \rightarrow \infty} q\left(x_{n}, x_{n+1}\right)=0 .
$$

Now we prove that $\left\{x_{n}\right\}$ is a left-Cauchy sequence in $(X, q)$. On contrary suppose that $\left\{x_{n}\right\}$ is not left Cauchy.

Then there exist an $\epsilon>0$ and monotone increasing sequences of natural numbers $\left\{m_{k}\right\}$ and $\left\{n_{k}\right\}$ such that $n_{k}>m_{k}$,

$$
q\left(x_{m_{k}}, x_{n_{k}}\right) \geq \epsilon
$$

and

$$
q\left(x_{m_{k}}, x_{n_{k}-1}\right)<\epsilon
$$

From (6) and (7), we obtain

$$
\begin{aligned}
\epsilon & \leq q\left(x_{m_{k}}, x_{n_{k}}\right) \\
& \leq q\left(x_{m_{k}}, x_{n_{k}-1}\right)+q\left(x_{n_{k}-1}, x_{n_{k}-1}\right)+q\left(x_{n_{k}-1}, x_{n_{k}}\right) \\
& <\epsilon+q\left(x_{n_{k}-1}, x_{n_{k}-1}\right)+q\left(x_{n_{k}-1}, x_{n_{k}}\right) .
\end{aligned}
$$

Letting $k \rightarrow \infty$ and then using (6), we get

$$
\lim _{k \rightarrow \infty} q\left(x_{m_{k}}, x_{n_{k}}\right)=\epsilon .
$$

Letting $k \rightarrow \infty$ and then using (5) and (8) in

$\left|q\left(x_{m_{k}-1}, x_{n_{k}}\right)-q\left(x_{m_{k}-1}, x_{m_{k}}\right)\right| \leq q\left(x_{m_{k}}, x_{n_{k}}\right)$

we obtain

$$
\lim _{k \rightarrow \infty} q\left(x_{m_{k}-1}, x_{n_{k}}\right)=\epsilon .
$$

Letting $k \rightarrow \infty$ and then using (5) and (8) in

$\left|q\left(x_{m_{k}}, x_{n_{k}+1}\right)-q\left(x_{n_{k}}, x_{n_{k}+1}\right)\right| \leq q\left(x_{m_{k}}, x_{n_{k}}\right)$

we obtain

$$
\lim _{k \rightarrow \infty} q\left(x_{m_{k}}, x_{n_{k}+1}\right)=\epsilon .
$$

Hence, we get

Since $f$ is weak triangular $\alpha$-admissible. Then, from Lemma 2.13 we have

$$
\alpha\left(x_{n_{k}}, x_{m_{k}}\right) \geq 1
$$


If $\frac{1}{2} q\left(x_{m_{k}-1}, x_{m_{k}}\right)>q\left(x_{m_{k}-1}, x_{n_{k}}\right)$ then letting $k \rightarrow \infty$,

we get $0 \geq \epsilon$ from 5 and 9 .

It is a contradiction. Hence

$\frac{1}{2} q\left(x_{m_{k}-1}, x_{m_{k}}\right) \leq q\left(x_{m_{k}-1}, x_{n_{k}}\right)$.

From (3), we have

$$
\begin{aligned}
& \psi\left(q\left(x_{m_{k}}, x_{n_{k}+1}\right)\right) \\
& \quad=\psi\left(q\left(f x_{m_{k}-1}, f x_{n_{k}}\right)\right) \\
& \quad \leq F\left(\psi\left(M\left(x_{m_{k}-1}, x_{n_{k}}\right)\right), \varphi\left(M\left(x_{m_{k}-1}, x_{n_{k}}\right)\right)\right),
\end{aligned}
$$

where

$$
M\left(x_{m_{k}-1}, x_{n_{k}}\right)=\max \left\{q\left(x_{m_{k}-1}, x_{n_{k}}\right), \frac{1+q\left(x_{m_{k}-1}, x_{m_{k}}\right) \cdot q\left(x_{n_{k}}, x_{n_{k}+1}\right)}{1+q\left(x_{m_{k}-1}, x_{n_{k}}\right)}\right\} .
$$

Letting $k \rightarrow \infty$ and then using (10) and (5) we have

$$
\begin{aligned}
\psi(\epsilon) & \leq F(\psi(\max \{\epsilon, 0\}), \varphi(\max \{\epsilon, 0\})) \\
& \leq F(\psi(\epsilon), \varphi(\epsilon)) .
\end{aligned}
$$

It follows that $\psi(\epsilon)=0$ or $\varphi(\epsilon)=0$. This implies that $\epsilon=0$ which is a contradiction. Hence $\left\{x_{n}\right\}$ is left Cauchy in $(X, q)$. Similarly, $\left\{x_{n}\right\}$ is right - Cauchy

Thus $\left\{x_{n}\right\}$ is a Cauchy sequence in $(X, q)$.

Hence,

$$
\lim _{m \rightarrow \infty} q\left(x_{n}, x_{m}\right)=0 .
$$

Since $x_{n+1}=f x_{n}$, it follows $\left\{x_{n}\right\}$ is a Cauchy sequence in the complete quasi - metric space $(X, q)$. Therefore, there exists $u \in X$ such that

$$
\lim _{n \rightarrow \infty} q\left(x_{n}, u\right)=\lim _{n \rightarrow \infty} q\left(u, x_{n}\right)=0 .
$$

From continuity of $f$ we get

$$
\lim _{n \rightarrow \infty} q\left(x_{n}, f u\right)=\lim _{n \rightarrow \infty} q\left(f x_{n-1}, f u\right)=0 .
$$

and

$$
\lim _{n \rightarrow \infty} q\left(f u, x_{n}\right)=\lim _{n \rightarrow \infty} q\left(f u, f x_{n-1}\right)=0 .
$$

Combining (13) and (14), we deduce

$$
\lim _{n \rightarrow \infty} q\left(x_{n}, f u\right)=\lim _{n \rightarrow \infty} q\left(f u, f x_{n}\right)=0 .
$$

From 12 and 15, due to the uniqueness of the limit, we conclude that $u=f u$, that is,$u$ is a fixed point of $f$.

Now we claim that, for each $n \geq 1$, at least one of the following assertions holds.

$$
\frac{1}{2} q\left(x_{n-1}, x_{n}\right) \leq q\left(x_{n-1}, u\right) \quad \text { or } \quad \frac{1}{2} q\left(x_{n}, x_{n+1}\right) \leq q\left(x_{n}, u\right) .
$$

On the contrary suppose that

$$
\frac{1}{2} q\left(x_{n-1}, x_{n}\right)>q\left(x_{n-1}, u\right) \text { and } \frac{1}{2} q\left(x_{n}, x_{n+1}\right)>q\left(x_{n}, u\right)
$$

for some $n \geq 1$.

Then we have

$$
\begin{aligned}
q\left(x_{n-1}, x_{n}\right) & \leq q\left(x_{n-1}, u\right)+q\left(u, x_{n}\right) \\
& <\frac{1}{2}\left[q\left(x_{n-1}, x_{n}\right)+q\left(x_{n}, x_{n+1}\right)\right] \\
& \leq q\left(x_{n-1}, x_{n}\right)
\end{aligned}
$$


which is a contradiction and so the claim holds.

Suppose $\frac{1}{2} q\left(x_{n}, x_{n+1}\right) \leq q\left(x_{n}, u\right)$.

Suppose $f u \neq u$.

Since the sequence $\left\{x_{n}\right\}$ converges to $u \in X$,from $(i i i)$, there exists a subsequence $\left\{x_{n}(k)\right\}$ of $x_{n}$ such that $\alpha\left(x_{n}(k), u\right) \geq 1$ and $\alpha\left(u, x_{n}(k)\right) \geq 1$ for all $k$.

We have

$$
\frac{1}{2} q\left(x_{n_{k}}, x_{n_{k}+1}\right) \leq \frac{1}{2} q\left(x_{n_{k}}, u\right)
$$

from (3), we have

$$
\psi\left(q\left(f x_{n_{k}}, f u\right)\right) \leq F\left(\psi\left(M\left(x_{n_{k}}, u\right)\right), \varphi\left(M\left(x_{n_{k}}, u\right)\right)\right),
$$

where

$$
M\left(x_{n_{k}}, u\right)=\max \left\{q\left(x_{n_{k}}, u\right), \frac{1+q\left(x_{n_{k}}, f x_{n_{k}}\right), q(u, f u)}{1+q\left(x_{n_{k}}, u\right)}\right\} .
$$

Letting $n \rightarrow \infty$ and using 14 , we get

$$
\begin{aligned}
& \psi(q(u, f u)) \\
& \quad \leq F\left(\psi\left(\max \left\{q(u, u), \frac{1+q(u, u) \cdot q(u, f u)}{1+q(u, u)}\right\}\right), \varphi\left(\max \left\{q(u, u), \frac{1+q(u, u) \cdot q(u, f u)}{1+q(u, u)}\right\}\right)\right) \\
& \quad \leq F(\psi(q(u, f u)), \varphi(q(u, f u)))<\psi(q(u, f u)),
\end{aligned}
$$

which is a contradiction.

Thus, $f u=u$.

Hence, $u$ is a fixed point of $f$.

$(H)$ for all $x, y \in F i x(f)$, we have $\alpha(x, y) \geq 1$, where Fix(f) denotes the set of fixed points of $f$.

Theorem 3.2. Adding $(H)$ to the hypotheses of Theorem (3.1), $f$ has a unique fixed point.

Proof. Due to Theorem (3.1), we have $u$ is a fixed point of $f$. Let $w$ be another fixed point of $f$.

Suppose $u \neq w$.

From $(H)$, we have

$$
\alpha(u, w) \geq 1, \text { for all } u, w \in F i x(f) .
$$

Since $\left.\frac{1}{2} q(u, f u)\right\} \leq q(u, w)$, from $(3)$, we obtain

$$
\begin{aligned}
\psi(q(u, w)) & =\psi(q(f u, f w)) \\
& \leq F(\psi(M(u, w)), \varphi(M(u, w)))
\end{aligned}
$$

where

$$
\begin{aligned}
M(u, w) & =\max \left\{q(u, w), \frac{1+q(u, u) \cdot q(w, w)}{1+q(u, w)}\right\} \\
& =q(u, w) .
\end{aligned}
$$

Thus

$\psi(q(u, w)) \leq F(\psi(q(u, w)), \varphi(q(u, w)))$.

It follows that $\psi(q(u, w))=0$ or $\varphi(q(u, w))=0$.

This implies that $q(u, w)=0$ which is a contradiction.

Hence $u=w$. 
Example 3.3. Let $X=[0, \infty)$ and $q$ be the quasi metric on $X$ given by

$$
q(x, y)=\left\{\begin{array}{cl}
|x| & \text { if } x \neq y \\
0 & \text { if } x=y
\end{array}\right.
$$

for all $x, y \in X$. It is obvious that $(X, q)$ be a complete quasi- metric space. Suppose that $f: X \rightarrow X$ is defined by

$$
f x=\left\{\begin{array}{cc}
x^{3}-2 x & \text { if } x>2, \\
\frac{x}{8} & \text { if } x \in[0,2] .
\end{array}\right.
$$

Now, define $\alpha: X \times X \rightarrow[0, \infty)$ as

$$
\alpha(x, y)=\left\{\begin{array}{cc}
1 & \text { if } x, y \in[0,1] \\
0 & \text { otherwise }
\end{array}\right.
$$

$$
\begin{aligned}
& \text { Let } F(s, t)=s-t \text { for all } s, t \in[0, \infty) \text {. Let } \psi(t)=t, \varphi(t)=\frac{t}{2} . \\
& \qquad \begin{aligned}
& \frac{1}{2} q(x, f x) \leq x \\
& \qquad(q(f x, f y))=q(f x, f y) \\
&=f x, \\
&=\frac{x}{8} \\
&=\frac{1}{2} q(x, y) \\
& \leq \frac{1}{2} M(x, y) \\
&=M(x, y)-\frac{1}{2} M(x, y) \\
&=F(\psi(M(x, y)), \varphi(M(x, y)))
\end{aligned}
\end{aligned}
$$

Therefore, all of the conditions of Theorem 3.1 are satisfied and 0 is the fixed point of $f$.

If we let $\alpha(x, y)=1$ for all $x \in X$, we get the following result.

Corollary 3.4. Let $(X, q)$ be a complete quasi metric space and let $f: X \rightarrow X$ be a given mapping $f$ is an $F(\psi-\phi)$-Suzuki- type rational contraction condition. If there exist functions $\psi \in \Psi, \varphi \in \Phi$ and $F \in \mathcal{C}$ such that

implies that

$$
\frac{1}{2} q(x, f x) \leq q(x, y)
$$

$$
\psi(q(f x, f y)) \leq F(\psi(M(x, y)), \varphi(M(x, y)))
$$

where

$$
M(x, y)=\max \left\{q(x, y), \frac{1+q(x, f x) \cdot q(y, f y)}{1+q(x, y)}\right\},
$$

for all $x, y$ in $X$. Then $f$ has a unique fixed point in $X$.

\section{References}

[1] A.H. Ansari, Note on $\phi-\psi$-contractive type mappings and related fixed point, The 2nd Regional Conference on Math.Appl.PNU, Sept.(2014), 377-380.

[2] S. Banach, Sur les operations dans les ensembles abstraits et leur application aux equation integrals, Fund. Math.,3(1922),133-181.

[3] E. Karapinar, B. Samet, Generalized $(\alpha-\psi)$ contractive type mappings and related fixed point theorems with applications. Abstr. Appl. Anal , 2012 (2012) Article id: 793486

[4] E. Karapinar, P. Kumam, Salimi, On $\alpha-\psi$ - Meir-Keeler contractive mappings, Fixed Point Theory Appl.2013, Article ID94(2013).

[5] O. Popescu, Two generalizations of some fixed point theorems, Comp. Math. Appl., 62, 3912-3919, (2011).

[6] B. Samet, C. Vetro, P. Vetro, Fixed point theorems for $\alpha-\psi$ - contractive mappings, Nonlinear Anal. 75(2012), $2154-2165$.

[7] T. Suzuki, A generalized Banach contraction principle which characterizes metric completeness, Proc. Amer. Math. Soc. 2008. vol. 136, pp. 1861-1869. 